Coalition for Diversity and Inclusion in Scholarly Communications • Antiracism Toolkit for Organizations

\title{
Introduction to the \\ Antiracism Toolkit for \\ Organizations
}

Toolkits for Equity in Scholarly Publishing Project Volunteers

Published on: Aug 25, 2021

DOI: 10.21428/77410d6b.5bc04707

License: Creative Commons Attribution-NonCommercial-ShareAlike 4.0 International

License (CC-BY-NC-SA 4.0). 
Building equity and promoting antiracism at organizations are not the jobs of specific individuals but are collective responsibilities. This toolkit, written by a multiracial group of industry professionals, is intended to help individuals at all levels within scholarly publishing organizations implement inclusive policies, procedures, and norms. The writers of this toolkit use the term "Black, Indigenous, and People of Color" and the acronym "BIPOC" to be as inclusive as possible. We have since become aware that the term "People of the Global Majority" is increasing in popularity [1].

Many characteristics of white supremacy culture-the ideology that white people and the ideas, thoughts, beliefs, and actions of white people are superior to Black, Indigenous, and People of Color (BIPOC) and their ideas, thoughts, beliefs, and actions -show up in our organizations. These damaging yet pervasive characteristics include perfectionism, paternalism, individualism, and a constant sense of urgency [2]. Some people may assume that these attributes are simply professional standards, with no connection to whiteness, and organizations may be unaware of their perpetuation of these characteristics; their racism may not be intentional. However, structural racism is built into our society, and it takes concentrated effort to recognize and address how and where racism and white supremacy show up in our organizations. Through this toolkit, we provide tools for organizations to understand institutionalized racism, to better support BIPOC staff, and to better serve BIPOC authors, readers, and reviewers.

\section{Key outcomes of using this toolkit:}

- Leaders and managers will be able to create a structure for diversity, equity, and inclusion (DEI) work; measure progress; ensure that antiracism work carries over into both distributed and co-located workforce environments; and assess how power and decision-making can be made more inclusive.

- Human resources departments will be able to broaden hiring and recruiting efforts; develop retention plans; establish equitable performance evaluation processes; and create Employee Resource Groups and mentorship programs.

- Staff in all departments and at all levels will learn to recognize and address bias; be conscious of practices that may unfairly tax BIPOC; and will be ready to take part in or lead affinity groups and mentorship programs.

\section{From Diversity to Antiracism}

To combat the systemic legal, social, and institutional constraints that have created barriers to racial equality, we must move beyond a diversity mindset-which may 
foster tokenism - to an actively antiracist mindset. What is the difference between "diversity" and "antiracism" in the workplace? Ibram X. Kendi defines an antiracist as:

One who is supporting an antiracist policy through their actions or expressing an antiracist idea...One who is expressing the idea that racial groups are equals and none needs developing, and is supporting policy that reduces racial inequity [ $\underline{3}]$.

An organization that is actively antiracist has policies and practices in place that address systemic inequities. Diversity, equity, and inclusion (DEI) efforts (which typically emphasize the D) can be a means to an antiracist end but often do not go far enough. In the scholarly publishing workplace, DEI initiatives may include optional trainings for staff, book discussions, and a presence at career fairs at Historically Black Colleges and Universities (HBCUs). In contrast, an antiracist workplace may eliminate unpaid internships (which preclude individuals from economically insecure backgrounds), mandate anti-bias training for hiring managers, and embed discussion around paths to advancement and promotion in the performance review process. We discuss these and other ideas in the pages that follow. While this toolkit uses the language of DEI, the approach taken here is an explicitly antiracist one-using the tools of DEI to advance policies that reduce racial inequity.

Scholar and educator Beverly Daniel Tatum uses the metaphor of a moving walkway at the airport to help people understand the ongoing cycle of racism:

Active racist behavior is equivalent to walking fast on the conveyor belt. The person engaged in active racist behavior has identified with the ideology of White supremacy and is moving with it. Passive racist behavior is equivalent to standing still on the walkway. No overt effort is being made, but the conveyor belt moves the bystanders along to the same destination as those who are actively walking. Some of the bystanders may feel the motion of the conveyor belt, see the active racist ahead of them, and choose to turn around, unwilling to go to the same destination as the White supremacists. But unless they are walking actively in the opposite direction at a speed faster than the conveyor belt - unless they are actively antiracist - they will find themselves carried along with the others [ $\underline{4}]$.

In order to disrupt the cycle of white supremacy in our workplaces, antiracist publishers must decenter whiteness and normalize the perspectives and experiences of BIPOC staff. To expand on the metaphor above, we should not only walk the antiracist walk as individuals, but also question the organizational structures that should be changed-the conveyor belt itself. Our Antiracism Toolkit for Allies [ $\underline{5}$ ] is a resource for individuals wishing to focus on their personal journey; this toolkit will focus on the work we can do to make our scholarly communications workplaces antiracist. 


\section{From Performative Activism to Sustained Actions}

I've seen these knee-jerk reactions... The knee eventually falls back into place.

- Charles Blow, columnist and lecturer, speaking at Springer Nature's Black Employment Network launch event []]

In the aftermath of high-profile events, such as the killing of George Floyd by Minneapolis police in 2020, many organizations responded with statements on DEI commitments, talks from notable Black commentators, reading lists for allies, and general affirmations of antiracism. Are these words motivated by a genuine recognition of white supremacy in society? Or is the organization merely responding to the event itself, including the threat that protest and civil unrest pose to corporate interests? Is it performative activism rather than a commitment to sustained action?

\section{Across racial, ethnic groups, more see pressure rather than genuine concern as contributing a great deal to companies' statements about race}

Among the $80 \%$ of U.S. adults who say they have seen or heard companies and organizations making public statements about race or racial inequality in the past few months, \% who say the following have contributed a great deal to these recent public statements

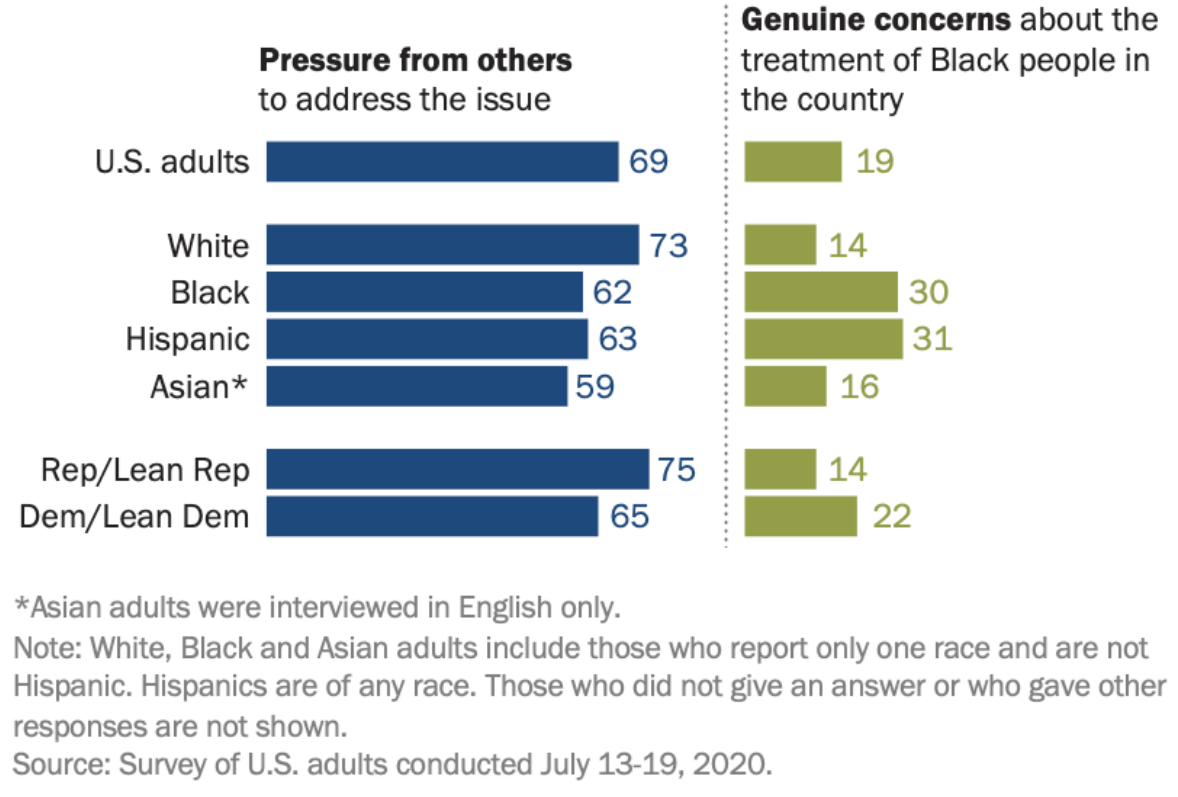

PEW RESEARCH CENTER

Credit: Pew Research Center, Washington, DC 
Even well-publicized examples of lethal racism only temporarily affect the attitudes of the majority []]. The occurrence of these critical moments can give the impression that racism is an episodic affair, a momentary deviation from an otherwise benign state of normality. But as J. Kēhaulani Kauanui observed regarding settler colonialism, racism is "a structure, not an event" []]. Racism, according to Eduardo Bonilla-Silva, refers to the social systems that distribute economic, political, social, and psychological rewards differently along racial lines, and where these historic and institutional differences have become normalized, an everyday feature of contemporary life [ㅁ] . Most racism exists below the surface, invisible to the majority of white people and organizations operating in predominantly white spaces, while BIPOC confront and live with these problems every day. Unless these events compel organizations to enact change and make a genuine commitment to DEI, they will stay in a reactive mode, responding to individual events rather than confronting systemic obstacles.

Tokenistic initiatives based on words alone inhibit the endeavor to address racism in the workplace. For example, organizations may make some effort to discuss DEI and organize gatherings for BIPOC employees, but as Charles Blow outlined: "On some level it is meant to make us happy and more docile and more quiet. 'Go in that room and talk it out. We'll send some donuts... and when you come out I hope you're happy and go back to work'” [10]. White people, meanwhile, may not move beyond the initial step of self-awareness and education to actually create a safer and more just work environment. As Ijeoma Oluo warned, "if your anti-racism work prioritizes the 'growth' and 'enlightenment' of white America over the safety, dignity and humanity of people of color-it's not anti-racism work. It's white supremacy" [11].

Absent an organizational commitment to action, disempowered BIPOC employees are confronted with extra work and only minimal efforts at inclusion, while white employees may think they are antiracist without having to back this up in a meaningful way. An organization's commitment to act can be seen through its commitment to change, and there is precedent for this kind of change in scholarly publishing.

Within scholarly publishing, one success story has been improving gender diversity at the leadership level [12]. If organizations want to take the fight against racism seriously, they will need to make similar commitments to recruiting, promoting, and retaining BIPOC. The starting point is clearly different: women make up more than half of the workforce in publishing [13], while Black people, for example, make up 5 percent or less [14],$[\underline{15}]$, [16]. Despite the differences between gender and race, organizations can apply similar methods, such as the use of transparent metrics and 
maturity models [17]. Specific models are available for developing antiracism in an organization (see the Continuum on Becoming an Anti-Racist Multicultural Organization [18]). This puts the development of an organization's DEI efforts on similar terms to individual development in becoming an antiracist. Once it is acknowledged that superficial acts are inadequate, the business must take responsibility for how to meet mature antiracist goals.
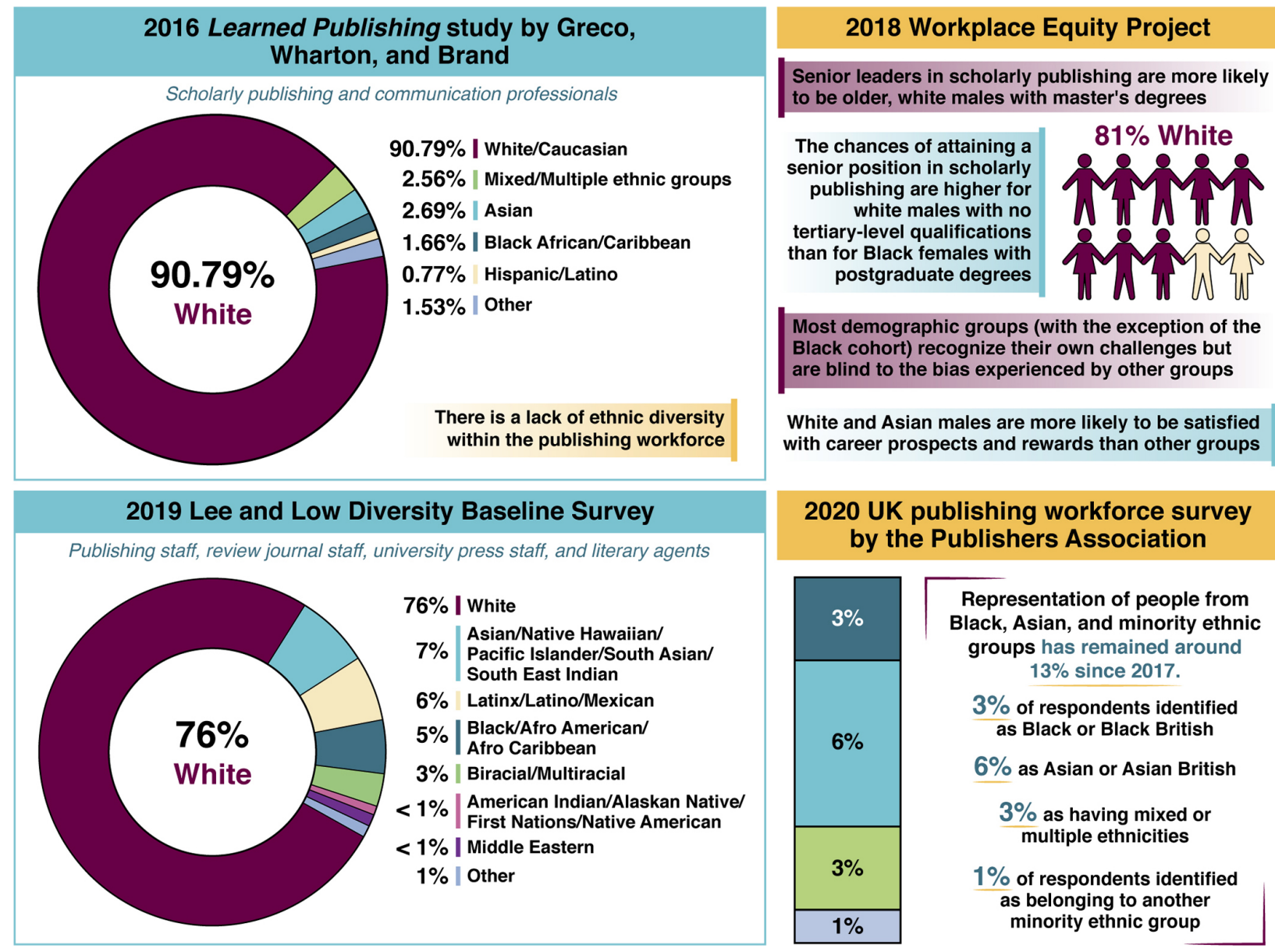

Credit: Greco, Wharton, and Brand; Workforce Equity Project; United Kingdom Publishers Association, and Lee and Low Diversity Baseline Survey.

\section{Prioritizing Equity}

It is tempting for organizations to take a utilitarian approach to the problem of racism. Case study after case study has shown the value that diversity brings to the work environment: diverse teams are more innovative, they help organizations gain understanding into more diverse markets, and they have a positive impact on the bottom line [19]. Foregrounding racial equity positions our organizations to better serve our constituencies; retain and attract a diverse staff; identify racial and ethnic 
disparities in research studies [20]; and avoid offensive missteps [21]], [22]. Yet, at many organizations, DEI leaders struggle for resources that would allow them to move past token inclusion toward true antiracist practices.

Organizations must look beyond the business case for diversity and inclusion and instead pursue practices and policies that prioritize racial equity because it is the ethical thing to do. In the Harvard Business Review, researchers Laura Morgan Roberts and Anthony J. Mayo argue:

[A racially just workplace] involves shifting from an exclusive focus on the business case for racial diversity to embracing the moral one, promoting real conversations about race, revamping diversity and inclusion programs, and better managing career development at every stage... The reward will be great: maximizing the human potential of everyone in the workplace [23].

Organizations that view antiracism as foundational to their mission will develop the staying power that the work requires. The deep-rooted inequities that exist throughout the scholarly communications ecosystem cannot be excised by a task force, a one-time budget allocation, or even the hiring of a DEI officer. Organizations that fully embrace antiracism, that embed it not just in their hiring practices, but also in their everyday work processes and strategies, will develop the muscles and skills needed for the long journey ahead. Racial equity work must be at the heart of everything we do to fully reach, serve, and represent the entire research community.

\section{Global Application}

Our antiracism framework is grounded in work by Allies for Change and the Racial Equity Institute, training organizations that are both based in the United States. The toolkit is intended primarily for publishing organizations in countries with a history of colonialism and settler colonialism, and with legacies of white supremacy, slavery, and Indigenous erasure. We hope that some of its content will be useful to readers around the globe-our CC-BY-NC-SA license allows it to be adapted by anyone-and that the toolkit will help equip all publishers to become better partners to readers, authors, reviewers, librarians, and the worldwide community of scholars.

This toolkit is focused on helping organizations transform their workplaces through antiracism. While this transformation will have ripple effects on how we do the work of publishing, we believe that the practical steps needed to transform our publishing practices and address the many inequities created by systemic racism and bias for our readers and researchers necessitate their own resources. In short, this guide will be useful for publishers implementing (or preparing to implement) organizational change, 
but it is only a small contribution to the groundwork that is needed to more fully transform the scholarly communications ecosystem.

\section{Antiracist Business Strategies}

According to the Harvard Business Review, anyone can use the antiracist business strategy expressed in the "four Ps":

Purchasing Power. Are the vendors that you work with diverse? Whom do you consider as packagers, printers, and freelancers? Make sure that you are paying freelancers fairly, based on average rates (see the rates outlined by the Editorial Freelancer's Association).

Philanthropy/Grant Programs. Commit to long-term support of local communities or charitable organizations by way of staff volunteers and financial assistance. Princeton University Press has created a Global Equity Grant program to provide funds for support to authors in categories such as family care, travel funds, translation, and media coaching.

Policy. Review internal and external systems to ensure equity. If you have an advocacy group, find out if there are policies that promote equity in your field that you can legally support.

Place. Are you investing in areas with majority minority populations or organizations that support marginalized individuals? Consider those in your own local community. Many organizations make donations to the American Civil Liberties Union (ACLU), the Equal Justice Initiative (EJI), and many others. If you are an engineering organization, for example, you can support the National Action Council for Minorities in Engineering (NACME).

You will also want to conduct a review of the systems in your organization. Are there recruitment, hiring, onboarding, and retention procedures and policies in place to give individuals what they need to be successful? Have they been reviewed through a DEI lens? If you are a membership organization, what do your demographic data look like? Who is included in your potential membership base? What does your board look like? What are the demographics of your staff, authors, readers, freelancers, and vendors? To whom are you reaching out and serving? Is diversity represented at all levels of your organization? Where are you looking for future board members? Who is being mentored to take on this role? 
Finally, encourage all employees to speak out against racism, sexism, ableism, ageism, or any other kind of discrimination and/or hate speech. Set up clear guidelines for colleagues to report these behaviors in a way that is sensitive, yet transparent and accountable. Your staff should feel comfortable raising instances that may be anything from systemic to another employee using microaggressions. Protect those who speak out and reevaluate your whistleblower policy regularly. Consider all cases no matter how small. Think of this feedback as an opportunity to improve. Ensure that there is a methodology in place to learn, grow, and correct your processes or culture once these grievances are made known.

\section{Citations}

1. https://regenerative.medium.com/im-embracing-the-term-people-of-the-globalmajority-abd1c1251241

2. Tema Okun, "White Supremacy Culture," dRworks, https://www.dismantlingracism.org/uploads/4/3/5/7/43579015/okun white sup_culture.pdf $\subseteq$

3. Kendi, Ibram X. How to Be an Antiracist. Random House. $2019 \subseteq$

4. Beverly Daniel Tatum, Why Are All the Black Kids Sitting Together in the Cafeteria? And Other Conversations About Race, 20th anniv. ed. (New York: Basic Books, 2017), 91.

5. https://c4disc.org/toolkits-for-equity $\perp \models$

6. Bynoe, Bernadette. Charles Blow Tells You How to Actually Fight

Racism. Scientific American, (https://www.scientificamerican.com/article/charlesblow-tells-you-how-to-actually-fight-racism/), $2021 \boxminus$

7. https://www.pewresearch.org/fact-tank/2020/09/16/support-for-black-lives-matterhas-decreased-since-june-but-remains-strong-among-black-americans/

8. J. Kēhaulani Kauanui, “'A Structure, Not an Event': Settler Colonialism and Enduring Indigeneity," Lateral: Journal of the Cultural Studies Association 5, no. 1 (2016), https://csalateral.org/issue/5-1/forum-alt-humanities-settler-colonialismenduring-indigeneity-kauanui/. 
9. Eduardo Bonilla-Silva, "Rethinking Racism: Toward a Structural Interpretation," American Sociological Review 62, no. 3 (1997): 465-480. 10. Blow, Charles. A conversation with Charles Blow (speech at Springer Nature Black Employee Network launch event). $2021=$ 11. Oluo, Ijeoma. Confronting racism is not about the needs and feelings of white people, (https://www.theguardian.com/commentisfree/2019/mar/28/confronting= racism-is-not-about-the-needs-and-feelings-of-white-people), 2019. 12. Jacobs, Rachel. Setting targets at Springer Nature to help create a better balance, (https://www.springernature.com/gp/advancingdiscovery/blog/blogposts/setting-targets-at-springer-nature-to-help-create-a-betterbalan/16531274), 2019.

13. Anderson, Porter. International Women's Day: PublisHer Issues a Diversity Toolkit, (https://publishingperspectives.com/2021/03/international-womens-day_ publisher-issues-diversity-inclusivity-toolkit-covid19/), 2021.

14. Jiménez, Laura, and Beckert, Betsy. Where Is The Diversity In Publishing? The 2019 Diversity Baseline Survey Results, (https://blog.leeandlow.com/2020/01/28/2019diversitybaselinesurvey/), 2020. 15. Greco, A. N., Wharton, R. M., \& Brand, A. (2016). Demographics of scholarly publishing and communication professionals. Learned Publishing, 29(2), 97-101. https://doi.org/10.1002/leap.1017 $\leftrightharpoons$

16. Taylor, S., Spilka, S., Monahan, K., Mulhern, I., \& Wachter, J. (2020). Evaluating equity in scholarly publishing. Learned Publishing, 33(4), 353-367. https://doi.org/10.1002/leap.1301

17. PublisHer. Diversity and Inclusion in the Publishing Industry: Diagnostic Toolkit Supporting Publishing Companies To Become More Diverse And Inclusive, (https://womeninpublishing.org/wp-content/uploads/2021/03/PublisHer-DiagnosticToolkit-EN.pdf), 2021.

18. Crossroads Ministry, Chicago, IL. Continuum on Becoming an Anti-Racist Multicultural Institution, (https://philanos.org/resources/Documents/Conference\%202020/PreRead\%20PDFs/Continuum AntiRacist.pdf), 2020. 
19. Sylvia Hewlett, Melinda Marshall, and Laura Sherbin, with Tara Gonsalves, Innovation, Diversity, and Market Growth (New York: Center for Talent Innovation, 2013); Sylvia Ann Hewlett, Melinda Marshall, and Laura Sherbin, "How Diversity Can Drive Innovation," Harvard Business Review, December 2013. https://hbr.org/2013/12/how-diversity-can-drive-innovation $\triangleq$

20. Lindsey Konkel, "Racial and Ethnic Disparities in Research Studies: The Challenge of Creating More Diverse Cohorts," Environmental Health Perspectives 123, no. 12 (2015): A297- A302, https://www.ncbi.nlm.nih.gov/pmc/articles/PMC4670264/.

21. https://www.marketwatch.com/story/jama-apologizes-and-deletes-tweet-thatquestioned-racism-in-medicine-11614889909

22. Candice Frederick, "Why Is Barnes and Noble Putting 'Literary Blackface' on Their Shelves?," Guardian, February 7, 2020, https://www.theguardian.com/commentisfree/2020 /feb/07/barnes-and-noble-diverseeditions-blackface. $\subseteq$

23. Toward a Racially Just Workplace. (2019, November 14). Harvard Business Review. https://hbr.org/2019/11/toward-a-racially_just-workplace $\triangleq$ 